\title{
El sueño de Von Neumann y la realidad de Freud
}

RESUMEN: Tomando como punto de partida lo que John von Neumann escribió en su libro El Ordenador y el cerebro y Sigmund Freud pensó y escribió en Proyecto de una psicología científica, se considera la cuestión de si el cerebro humano se puede comprender en base (o reducir) a la física y la matemática tal y como entendemos en la actualidad. Para ello se presta especial atención a cuestiones como: "conducta versus actividad", límites de la matemática (Gödel y Turing), explicaciones no algorítmicas, nuevas propuestas como la de Roger Penrose, y sistemas no lineales.

PALABRAS CLAVE: Cerebro, física y matemáticas, reduccionismo
SUMMARY: Taking as starting point what John von Neumann wrote in his book, Computer and the Brain, and what Sigmun Freud thought and wrote in his Project for a Scientific Psychology, the question of whether the human brain can be understood (or reduced) on the basis of physics and mathematics as they are understood presently, in considered. To do that special attention is paid to question such as: "conduct versus activity". Limits in mathematics (Gödel and Turing), nonalgorithmical explanations, new proposals lije that of Roger Penrose, and no lineal systems.

KEY WORDS: Brain, physics and mathematics, reduccionism.

\section{El sueño (reduccionista) de John von Neumann: el cerebro como un ordenador}

John von Neumann (1903-1957), un húngaro que terminó afincado en Estados Unidos, como miembro del Institute for Advanced Study de Princeton, fue uno de los mayores genios matemáticos del siglo XX, aunque calificarle simplemente de "genio matemático" sería desvirtuar la esencia y amplitud de su obra, ya que además de a la matemática "pura" (a ramas de ésta como el álgebra, espacios funcionales o geometría contínua) realizó contribuciones fundamentales a la matemática aplicada, física teórica y matemática, ciencias de la computación o economía (con su teoría de juegos). Pues bien, hacia el final de su poco dilatada vida, a principios de 1955, von Neumann fue invitado por la Universidad de Yale a dictar las prestigiosas Conferencias Silliman, las correspondientes a la primavera de 1956. Aceptó, eligiendo como tema "El ordenador y el cerebro". Escasos meses después de haber aceptado la invitación, en agosto de 1955, le fue diagnosticado un cáncer. No abandonó, sin embargo, el proyecto de las conferencias, continuando trabajando en el manuscrito. El cáncer, no obstante, siguió progresando (se le localizaron varias ramificaciones en la espina dorsal) y finalmente, confinado ya a una silla de ruedas desde enero de 1956, tuvo que desistir de la idea de dictar las 
conferencias, aunque fuera una sola. A pesar de ello, la Universidad de Yale no suprimió las conferencias, sugiriendo que si von Neumann podía entregar un manuscrito alguien lo leería en su lugar. Esto le animó a proseguir con su escritura, que, según dicen, continuó, aunque muy débilmente, incluso cuando en abril de 1956 ingresó en el hospital del que no saldría hasta su muerte, acaecida el 8 de febrero de 1957. No pudo, en cualquier caso, completarlo, pero incompleto fue publicado en 1958 con el título de The Computer and the Brain (El ordenador y el cerebro).

Incompleto como es, la lectura del texto de von Neumann no deja mucho lugar a dudas de que su propósito en el texto que deseaba completar era el que muchos otros, antes y después que él, se han planteado: en qué medida se parece el cerebro humano a un ordenador, cuestión que conduce inevitablemente a la de si es posible, al menos en principio, construir un ordenador que reproduzca las principales características de los humanos, de la mente, del cerebro, de los humanos, claro. ${ }^{1}$ Unas pocas citas extraídas del texto de von Neumann pueden servir para comprender que en última instancia tales eran las ideas del matemático magiar. Así, leemos (1):

"La observación más inmediata con respecto al sistema nervioso es que su funcionamiento es, prima facie, digital [digital debido a que los impulsos nerviosos pueden ser considerados como un marcador binario: la ausencia de un impulso representa, por ejemplo, el valor $0 \mathrm{y}$ su presencia el 1].

El componente básico de este sistema es la célula nerviosa, la neurona, y la función normal de una neurona es generar y propagar un impulso nervioso. Este impulso es un proceso más bien complejo, con una variedad de aspectos eléctricos, químicos y mecánicos. Parece, sin embargo, que constituye un proceso definido, es decir, es casi el mismo bajo cualesquiera condiciones, y representa una respuesta unitaria, esencialmente reproducible, a una variedad amplia de estímulos".

Y más adelante, añadía (2): “[Estas] observaciones muestran que el sistema nervioso, cuando se le considera como un autómata, debe tener tanto una parte aritmética como una parte lógica, y que las necesidades de aritmética son tan importantes como las de lógica. Esto significa que estamos de nuevo tratando con una máquina de calcular en el sentido estricto y que resulta apropiado un análisis en términos de los conceptos familiares de las máquinas de calcular".

En el sueño, o programa, de von Neumann primaban fundamentalmente los aspectos matemáticos: eran la lógica (el dominio de las comunicaciones de órdenes) y la aritmética (el de las comunicaciones de números) los protagonistas principales de su análisis, que incluso aplicaba al lenguaje. Y aunque reconocía que "la lógica y las matemáticas en el sistema nervioso central, cuando se las considera como lenguajes, deben ser estructuralmente distintas de aquellos lenguajes a los que se refiere nuestra experiencia corriente" ("idiomas como el griego o el sánscrito", afirmaba, "son realidades históricas y no necesidades lógicas"), terminaba manifestando -así acaba de hecho el libro- que "las anteriores observaciones acerca de la fiabilidad y la profundidad lógica y aritmética, prueban que, de cualquier forma que esté configurado el sistema [nervioso central], no puede diferir considerablemente de lo que consciente y explícitamente consideramos como matemáticas" (3). 
Parece claro que el sueño de von Neumann era un sueño reduccionista, un sueño, un programa, que buscaba explicar la realidad cerebral y mental en base a elementos específicos y a reglas de relaciones entre ellos claramente definidas. En ese sentido, no difería sustancialmente (aunque sí, evidentemente, en contenidos) de los programas científicos que, en la matemática, física, química y fisiología, habían ido estableciéndose desde Euclides (matemática), la Revolución Científica de los siglos XVI y XVII (física y química) o el siglo XIX (fisiología). Animado por los éxitos que había obtenido con computadores como ENIAC (Electronic Numerical Integrator And Computer), el primer computador electrónico digital (de cuyo diseño fueron responsables Presper Eckert y John Mauchly, pero a cuyo desarrollo el matemático húngaro prestó notables servicios), von Neumann se dispuso a adentrarse en los sin duda más complejos mundos del cerebro. No le dio tiempo -es dudoso en cualquier caso de que hubiese sido capaz- de plantearse problemas del tipo de si su enfoque podría acoger manifestaciones cerebrales y mentales como son, por ejemplo, las emociones, los sueños o las fobias; si habría sido, en definitiva, capaz de preguntarse en qué medida su aproximación podría dar cabida a los subterráneos universos con los que médicos e investigadores pugnaban desde hacía bastante, intentado proporcionarles también un marco teórico.

\section{El neurocientífico (reduccionista) Sigmund Freud}

Pero si de sueños hablamos, de sueños reduccionistas, hay que reconocer, como ya he mencionado, que otros, además de von Neumann ,también se los plantearon, aunque fuese desde ámbitos diferentes al de éste, en el que la matemática desempeñaba un papel central. Uno de ellos fue nada más y nada menos que Sigmund Freud, antes de que se convirtiese en el fundador del psicoanálisis.

Una primera evidencia de que Freud también fue susceptible a sueños reduccionistas, aunque no se manifestasen éstos en la, todavía lejana idea, de ordenadores, sino en las posibilidades de la fisiología (hoy hablaríamos de neurociencias), es lo que escribió el 20 de octubre de 1895 a su entonces íntimo amigo, Wilhelm Fliess Fliess (4):

"En una activa noche de la semana pasada, con ese grado de molestia dolorosa que establece el óptimo para mi actividad cerebral, se alzaron de pronto las barreras, cayeron los velos y se pudo penetrar con la mirada desde los detalles de las neurosis hasta las condiciones de la conciencia. Todo parecía ensamblarse, el engranaje encajaba y se tenía la impresión de que ahora la cosa era en verdad una máquina, que pronto empezaría a andar por sí sola.

Los tres sistemas de neuronas, el estado libre y ligado de cantidad, el proceso primario y el secundario, la tendencia principal y la de compromiso del sistema nervioso, las dos reglas biológicas de la atención y de la defensa, los signos de cualidad, realidad y pensamiento, el estado del grupo psicosexual -el condicionamiento de la represión por la sexualidad, finalmente las condiciones de la conciencia como función receptiva-, ¡todo esto concordaba y sigue concordando!". 
En otras palabras, Freud estaba expresando su convencimiento de que creía posible entender la psique humana y sus distintos trastornos en términos puramente fisiológicos, que lo mental podía reducirse a lo físico, que los procesos psíquicos debían ser representados como estados de, en última instancia, "partículas" materiales. Era, en este sentido, un hombre de su tiempo: recordemos que la psicología de entonces estaba dominada por la denominada psicología fisiológica o experimental, con Wilhelm Wundt, Ernst Weber y Gustav Theodor Fechner como sus principales representantes. Era aquella psicología una que trataba de reducir los procesos mentales o psíquicos a procesos fisiológicos, concretamente de los órganos de los sentidos y del cerebro.

El mismo año en que escribía a Fliess, Freud esbozó su visión de la mente basada en la fisiología en un manuscrito que posteriormente se llamaría Proyecto de una psicología científica, y que comienza con las siguientes palabras (5): "La finalidad de este proyecto es la de estructurar una psicología que sea una ciencia natural; es decir, representar los procesos psíquicos como estados cuantitativamente determinados de partículas materiales especificables, dando así a esos procesos un carácter concreto e inequívoco".

\section{La realidad de Freud: conducta versus actividad}

Su sueño fisiológico-reduccionista no duró, sin embargo, mucho, ya que cada vez se hizo más escéptico sobre la posibilidad de que la mente y sus trastornos se pudiesen explicar en términos fisiológicos. De hecho, no publicó el libro que estaba preparando, y cuando finalmente apareció, en 1950, con su autor ya hacía mucho muerto, lo hizo precedido por una "Advertencia a la edición alemana", añadida por los editores, en la que se lee (6): "Años después [Freud] aludió al fracaso de sus esfuerzos en este sentido [reducir la psiquiatría a la fisiología] con las siguientes palabras: 'La investigación científica ha demostrado irrebatiblemente que la actividad psíquica está vinculada a la función del cerebro más que a la de ningún otro órgano. La comprobación de la desigual importancia que tienen las distintas partes del cerebro y de sus relaciones particulares con determinadas partes del cuerpo y con determinadas actividades psíquicas nos lleva un paso más adelante, aunque no podríamos decir si este paso es grande. Pero todos los intentos realizados para deducir de estos hechos una localización de los procesos psíquicos, es decir, todos los intentos de concebir las ideas como almacenadas en las células nerviosas y las excitaciones como siguiendo el curso de las fibras nerviosas, han fracasado por completo"'.

Por haber llegado a esta convicción, por terminar creyendo que la realidad psíquica no se dejaba englobar en teorías reduccionistas basadas en términos puramente fisiológicos, una realidad de la que él era maestro consumado, a pesar de todas las deformaciones y mentiras que, como se ha ido sabiendo durante los últimos años, introdujo frecuentemente en sus presentaciones y reconstrucciones (7); por todo eso, aparece en el título de este artículo la expresión "la realidad de Freud": "El sueño de von Neumann", que fue también en cierto momento el de Freud, y "la realidad de Freud". 
Los motivos por los que Freud abandonó un camino y tomó otro constituyen una larga, personal e idiosincrática historia, que no me interesa especialmente ahora (8). Lo que sí me interesan son las razones que pueden justificar abandonar un enfoque reduccionista para intentar explicar "la naturaleza humana", expresión con la que quiero significar no sólo su fisiología, metabolismo y funciones similares, sino también su conducta, sana o enferma (si fuera únicamente lo primero -fisiología, metabolismo, etc.sería una pobre "naturaleza humana". Y para esta tarea de comprensión intelectual, que es al mismo tiempo la de combatir un reduccionismo grosero y primitivo, no he encontrado mejor ayuda que los escritos de Carlos Castilla del Pino.

Freud, como Castilla del Pino y tantos otros, buscaba comprender, sin duda, el -permítaseme utilizar por un momento este término tan oscuro- "alma" humana, pero ese alma, ese espíritu, ese "ser" no eran, no son, abstractos, se incardinaban, se incardinan, en personas concretas, con sus filias y sus fobias, sus virtudes y sus defectos, sus obsesiones o sus desprendimientos. El psiquiatra, en definitiva, utilizando la palabra de Castilla del Pino, "se encuentra, lo quiera o no, ante sujetos, ante individuos, a los cuales hay que diagnosticar, llegado el caso, una determinada enfermedad mental, pero a los cuales hay que atender en sus aspectos personales, en suma, biográficos. El propio Kräepelin, en su último trabajo, Las formas de manifestación de las enfermedades mentales, publicado poco antes de su muerte (1926), reconoce, en una rectificación que le honra, que la sintomatología de los enfermos mentales tiene tanto de perturbación funcional como de referencia biográfica, es decir, personal" (9).

Podemos pensar que el cerebro es el centro vital de los humanos, que toda actividad y decisión que les atañe pasa por ese universo de neuronas e interconexiones químicas y eléctricas, pero resulta que hay más, mucho más, desgraciada o afortunadamente (más esto que aquello: de nuevo, ¿qué humanos seríamos en caso contrario?). El cerebro rige -aceptemos esto en principio- nuestros movimientos, nuestras actividades, pero está también la conducta, que no es lo mismo. Recurramos, de nuevo, a Castilla del Pino (10):

"La conducta, el conjunto de actos por antonomasia, particularizados en actuaciones de sentido, debe diferenciarse de la actividad. Una actividad tal como respirar, bostezar, digerir, etc., y desde luego las más elementales de la contracción muscular, el intercambio $\mathrm{O}_{2} / \mathrm{CO}_{2}$, la agregación plaquetaria, etc., no son conductas. Son actividades biológicas que mantienen el organismo en condiciones homeostáticas tales como para hacer posible, luego, la conducta por parte del sistema-sujeto. La actividad (biológica) tiene un carácter molecular, a diferencia de la conducta que la tiene molar. La actividad biológica, que no se diferencia de la fisicoquímica en general más que en el hecho de que es una fisicoquímica de lo viviente, es condición necesaria para que la conducta sea posible, de la misma manera que la actividad fisicoquímica es condición necesaria para la actividad biológica. Se ha hablado impropiamente de 'biología de la conducta': con ello no pueden dilucidarse sino las actividades biológicas 
que hacen posible un acto, o muchos actos, de conducta; así, por ejemplo, la contracción muscular y el juego de articulaciones del brazo, antebrazo y mano hace posible que con el brazo se haga la señal de detener el autobús o la del saludo fascista, pero sería no pertinente hablar aquí de biología de una u otra conducta por el hecho de que sepamos con detalle el mecanismo neuroosteomuscular que subyace. Lo que interesa aquí, para inteligir la conducta, es su carácter de signo y su pertenencia a un código de señales, lo cual concierne a la Semántica y a la Semiología. No se puede, pues, identificar el mecanismo que hace posible la conducta con la conducta en sí misma: ni las cuerdas vocales son el habla ni el circuito del fórnix es la agresión, pero de ambos se infiere que podemos hablar y podemos agredir".

Los humanos son, ciertamente, biología, pero también cultura, relación con otros, y esto implica que no es posible establecer una frontera nítida entre la unidad biológica "ser humano" individual y el mundo. Por ello, como resalta Castilla (es la última cita que tomo de él, esta vez de su libro Un estudio sobre la depresión) (11): “el planteamiento positivista es ciertamente útil, tiene su misión, su objeto epistemológico es la dilucidación de la naturaleza de los procesos mentales, normales o patológicos; pero no es suficiente para dar cuenta de los problemas que conciernen al objeto psiquiátrico en su vertiente no natural sino cultural, que es siempre un sujeto... Mientras por su naturaleza la alteración mental ofrece síntomas en la esfera natural, y son modificaciones patológicas de actividades fisiológicas, el hecho de que sean perturbaciones del sujeto da lugar a modificaciones de la conducta, esto es, de los actos (psicologicosociales, culturales) o, más precisamente, de las actuaciones. Los actos que denominamos mentales son actos de conducta, como decir algo a alguien, sumar o restar, o soñar, o fantasear, o pensar en un problema, o recordar un acontecimiento, etc.; en suma, lo que se denomina actos intencionales o significativos. Los conductistas sembraron la confusión, al tiempo que se limitaron ostensiblemente pero voluntariamente como psicólogos, cuando consideraron sin más conductas tanto las actividades como los actos". Tras lo cual concluía:

"Ese reduccionismo a ultranza, disfrazado de ciencia actual, ha tenido sus seducciones, pero se agostó rápidamente, si bien representó en alguna medida una cierta disciplina, la disciplina de los hechos, a veces olvidados".

Un problema añadido para el sueño reduccionista: Gödel, Turing y los límites de los algoritmos

Vimos antes que en los esfuerzos y planteamientos de John von Neumann ocupaba un lugar fundamental la matemática, la idea de que la lógica y la matemática son elementos básicos para entender el cerebro y sus múltiples funciones. Sin embargo, en los últimos años un resultado matemático (que sin duda conocía von Neumann, pero que nunca utilizó en el sentido que veremos) ha suscitado vivas discusiones acerca de si el "reduccionismo matemático" es viable en el estudio de la mente humana. Me estoy refiriendo al teorema de incompletud desarrollado por Kurt Gödel en 1931, según el cual una teoría aritmética no puede ser a la vez consistente, axiomatizable y completa a 
la vez. Más concretamente, lo que Gödel demostró es que sistema formales como la aritmética elemental, al igual que cualquier otra teoría matemática que la contenga (lo que significa teorías como el análisis, cálculo vectorial o teoría de conjuntos), deben contener enunciados que no son demostrables ni indemostrables con los medios permitidos por el sistema (12).

Esta limitación lógica intrínseca fue tomada por el físico matemático Roger Penrose, en un libro de gran éxito, The Emperor's New Mind (La nueva mente del emperador; 1989), como evidencia de que "el concepto de verdad matemática no puede ser encapsulado en ningún esquema formalista", que la "noción de verdad matemática va más allá del concepto global de formalismo", que "hay algo absoluto e 'infuso' en la verdad matemática", que "la verdad matemática real va más allá de las simples construcciones humanas" (13).

Si este punto de vista fuese correcto, las consecuencias para el reduccionismo científico serían dramáticas, al menos entendiendo por "reduccionismo científico" el basado en los enfoques, como el de von Neumann, en los que la matemática "tradicional" desempeña un papel central, más aún si tenemos en cuenta los límites (otra manifestación del resultado de Gödel) con los que se topan las máquinas de Turing, el "computador idealizado" en el que se basan los ordenadores actuales, introducido por Alan Turing en 1937 (14). Para explicar cual es el problema en este caso, no resisto la tentación de utilizar lo que Jesús Mosterín escribió al respecto no hace mucho en su libro Ciencia viva (15): "Todos somos testigos de los enormes progresos de la computación en nuestro tiempo. Sin embargo, también ésta se topa con límites infranqueables. Todo lo que pueda hacer un computador posible de cualquier tipo lo puede hacer una máquina de Turing... Pues bien, el mismo Turing probó que no es posible construir una máquina de Turing que decida si un programa cualquiera dado puede ser ejecutado en un número finito de pasos por una máquina de Turing cualquiera dada. En general, la prueba de que una máquina de Turing (que, como computadora real, siempre sería muy lenta e ineficiente) puede computar algo carece de interés. Sin embargo, probar que la máquina de Turing no puede hacer algo es sumamente interesante, pues implica que nunca computador alguno podrá hacerlo, por mucha tecnología, dinero e inteligencia de que dispongamos. La incomputabilidad de Turing es siempre incomputabilidad por principio, incomputabilidad insuperable".

Decía que si fuese correcto el punto de vista de Penrose (y en última instancia, como acabamos de ver del propio Turing, aunque éste no llegase a formularlo así), según el cual "la verdad matemática va más allá de las simples construcciones humanas", entonces difícilmente podríamos evitar concluir que el reduccionismo matemático no permitirá acoger bajo su protector y acogedor manto a aquellas disciplinas que se ocupan de la mente humana, la psicología y la psiquiatría entre ellas, una conclusión que tal vez habría que tomar como constatación de la existencia de una barrera definida e infranqueable entre ambas y las neurociencias, conclusión que reforzaría las ideas expresadas en las dos secciones precedentes. 
De hecho, la incapacidad de una máquina de Turing para "computar todo" es una manifestación de los límites de los algoritmos. Parece obvio, en consecuencia, concluir que tal camino conduce directamente a pensar que una solución posible para encontrar una explicación, desde la ciencia, de cómo funciona la mente, de, por ejemplo, la conciencia, tendría que basarse en formulaciones no algorítmicas (sabemos que existen matemáticas no recursivas, esto es, matemáticas en las que aparecen problemas para los que no existe ningún algoritmo que permita contestar sí o no a una pregunta): "una buena parte de la razón", manifiesta Penrose, "para creer que la consciencia es capaz de influir en los juicios de verdad de una manera no-algorítmica deriva de la consideración del teorema de Gödel. Si podemos ver que el papel de la consciencia es no-algorítmico cuando formamos juicios matemáticos, en los que el cálculo y la demostración rigurosa constituyen un factor tan importante, entonces ciertamente podemos persuadirnos de que un ingrediente no-algorítmico semejante podría ser también crucial para el papel de la consciencia en circunstancias más generales (no matemáticas)”(16). ¿No será uno de esos "ingredientes no algorítmicos" la conducta?

No se limitó Penrose a las anteriores consideraciones, de carácter más o menos general, sino que intentó encontrar soluciones, en la mejor tradición reduccionista, aunque ahora con elementos diferentes, no algorítmicos, no, en este sentido, reduccionistas "clásicos": su sugerencia es que la solución provendrá de la física. Ahora bien, un problema no menor es que sus argumentos se basan en un nuevo tipo de teoría física todavía sin desarrollar. Su propuesta es que la clave para la comprensión de la concienciaconsciencia podría encontrarse en una teoría que reconcilie la teoría cuántica con la teoría general de la relatividad, que efectos gravitatorios aún no comprendidos podrían ser responsables del colapso de la función de onda cuántica, lo que aportaría un elemento no algorítmico a las leyes de la naturaleza.

No es excesivamente sorprendente que un físico haga una sugerencia de este tipo: casi todas las propuestas que existen relativas a la utilización de la física para explicar la conciencia se concentran en la mecánica cuántica, en la que, como vimos en el capítulo 1, se recurre a un mal definido "observador" como responsable del colapso de la función de onda. Es oportuno recordar en este punto que fue también von Neumann uno de los primeros en iniciar esta corriente de pensamiento, en su obra capital, Fundamentos matemáticos de la mecánica cuántica (1932), en la que escribió (17): "En primer lugar es en sí y de por sí absolutamente cierto que el medir y el proceso de la apercepción subjetiva con él ligado son algo nuevo respecto del mundo físico en torno, irreductible a éste. Es algo, en efecto, que nos lleva fuera de él, o más exactamente: nos conduce a dentro de la vida intelectual íntima del individuo, incontrastable en cuanto supuesto previo de todo intento de contrastación"; añadiendo poco después (18): "por lejos que llevemos los cálculos, hasta el depósito de mercurio, hasta la escala del termómetro, hasta la retina o el cerebro, llega un momento en que hay que decir: esto es percibido por el observador. Con otras palabras, siempre hemos de dividir el Universo en dos partes: una es el sistema observado, la otra el observador. En la primera podemos seguir todos los procesos físicos con tanta precisión como queramos (por lo menos en principio), en la última esto carece de sentido". 
Si nos fijamos, estas manifestaciones no son básicamente diferentes de las interpretaciones del teorema de Gödel en el sentido de que ponen un límite a la capacidad humana para interpretar en términos científicos la realidad de la naturaleza, lo que ocurre es que ahora el límite es uno impuesto por una teoría física, la mecánica cuántica, no por uno lógico.

El que Penrose proponga que puede existir una solución a ese problema, y que tal solución debe proceder de una nueva teoría que combine la mecánica cuántica y la teoría de la relatividad general es hasta cierto punto natural, ya que dentro de la interpretación de Copenhague de la teoría cuántica no parece existir salida al problema, por lo que es preciso ir más allá de esa teoría, y resulta que uno de los problemas abiertos clásicos de la física es construir una teoría cuántica de la relatividad general. El hecho de que exista un elemento no algorítmico, no reducible a cálculos, en el colapso de la función de onda podría -tal es la argumentación de Penrose- explicar ciertos aspectos de nuestra comprensión matemática, que, de nuevo según el físico matemático inglés, van más allá de la capacidad de cualquier sistema algorítmico, y en este sentido ayudar a explicar algunos elementos del funcionamiento cognitivo humano.

Como es natural, las propuestas de Penrose han sido criticadas por muchos. Uno de ellos es David Chalmers, quien ha escrito en uno de sus últimos libros (19):

"La conexión más frecuentemente mencionada entre la conciencia y la mecánica cuántica se encuentra en el hecho de que en algunas interpretaciones de esta última, se necesita la medición de un observador consciente para producir el colapso de la función de onda. En este tipo de interpretación, la conciencia tiene un papel central en la dinámica de la teoría física. Estas interpretaciones son sumamente controversiales, pero de cualquier forma es notorio que no hacen nada para proporcionar una explicación de la conciencia. Más bien, simplemente suponen la existencia de la conciencia, y la utilizan para ayudar a explicar ciertos fenómenos físicos....

No podemos descartar la posibilidad de que teorías físicas fundamentales como la mecánica cuántica desempeñen un papel clave en una teoría de la conciencia... Pero, de cualquier forma, hay pocas esperanzas de que este tipo de teorías proporcione una explicación totalmente física de la conciencia. Cuando se trata de explicación reductiva, las teorías físicas basadas en la física no están en una mejor situación que las teorías neurobiológicas o cognitivas".

Un intento de auxilio a las ideas de Penrose, desde la neurología, son los trabajos de Stuart Hameroff, del Departamento de Anestesiología y Psicología y del Centro de Estudios de la Consciencia de la Universidad de Arizona, que ha propuesto que la cognición humana podría depender de colapsos cuánticos en microtúbulos, estructuras proteínicas que se encuentran en el sistema de sostén de una neurona. Ambos, Penrose y Hameroff, han colaborado, sugiriendo que el colapso cuántico en microtúbulos podría ser la base física de la experiencia consciente (20). Pero no es preciso continuar por este camino: ni se ha probado nada, ni es de esperar que enfoques que tratan, de una forma $\mathrm{u}$ otra, de reducir la mente, la conciencia o consciencia, el, en definitiva, comportamiento humano, a la física tengan, puedan tener alguna vez éxito. Se necesitará, creo, otro tipo de explicación científica. 
NOTAS:

${ }^{1}$ No ignoro que, como veremos más adelante, en otro trabajo suyo (el libro que dedicó a los fundamentos de la mecánica cuántica) von Neumann planteó problemas sobre la problemática relación de física y consciencia. En cualquier caso, ni von Neumann llegó a pronunciarse sobre estas cuestiones, ni, en última instancia, importa demasiado que el papel que le otorgó aquí de adalid del reduccionismo, acaso no se corresponda a sus opiniones profundas. Siempre se le puede considerar como un tipo de metáfora. O que una vez, cuando su vida se apagaba, tuvo sueños reduccionistas.

\section{BIBLIOGRAFÍA}

(1) John von Neumann, El ordenador y el cerebro (Antoni Bosch, Barcelona 1980), pp. 59-60.

(2) Ibíd., pp. 82-83

(3) Ibíd., pp. 86-87

(4) Reproducida en Nicolás Caparros Sánchez, comp., Edición crítica de la correspondencia de Freud, tomo II 1887-1909 El descubrimiento del inconsciente Quipu Ediciones, Madrid 1995, p. 144

(5) Sigmun Freud, Proyecto de una psicología para neurólogos (1950). Incluido en Sigmund Freud, Obras completas. Tomo I 1873-1899 Biblioteca Nueva, Madrid 1997, traducción de Luis López-Ballesteros y de Torres; cita en la p. 211. Análisis específicos de esta obra de Freud, y su actualidad un siglo después, se encuentra en Neuroscience of the Centenal of Freud's 'Project for a Scientific Psychology', Robert M. Bilder y F. Frank LeFever, eds., Annaks of the New Cork

(6) S. Freud, Proyecto de una psicología para neurólogos, op. Cit., pp. 209-210.

(7) Ver en sentido, por ejemplo, Louis Breger, Freud, el genio y sus sombras, Javier Vergara Editor, Barcelona 2001.

(8) Algunos detalles se explican en Marck Sloms, "Befote and alter Freud's Project", en Neuroscience of the Mind, op. Cit., 1-10.

(9) C. Castilla del pino, "Prólogo" a Sigmun Freud, La interpretación de los sueños, Círculo de Lectores. Barcelona 1995, pp. 19-20.

(10) C. Castilla del Pino, "Epistemología de la psico(pato)logía: la conducta, relación sujeto/objeto", en Epistemología y práctica psiquiátrica, Manuel Desviat, coord. Asociación Española de Neuropsiquiatría, Madrid 1990., pp 105-134; pp. 114-115.

(11) C. Castilla de Pino, Un estudio sobre la depresión, Península, Barcelona 2002; primera edición de 1966. La cita está tomada del prefacio. "Este libro, hoy (o veinticinco años después). Otoño de 1990", pp. 15-16.

(12) K. Gödel, "Ubre formal unentscheidbare Sätze per Principia Matemática verwandter Systeme I”, Monatshefterfür Mathematik und Physik 38, 173-198 (1931). Existe versión al castellano en: K. Gödel, Obras completas (Alianza, Madrid 1987)

(13) Roger Penrose, La nueva mente del emperador (Mondador, Madrid 1991), pp. 152-153.

(14) A. Turing, "On computable numbers, with an application to the Entscheindunsproblem", Proceeding of the London Mathematical Society 42, 230-265 (1937).

(15) Jesús Mosterín, Ciencia viva, Espasa, Madrid 2001, p.92.

(16) R. Pensore, La nueva mente del emperador, op.cit., p. 516.

(17) John von Neumann, Fundamentos matemáticos de la mecánica cuántica (Consejo Superior de Investigaciones Científicas, Madrid 1991, p. 298.

(18) Ibíd., p. 299 
DEBATES

(19) David J. Chalmers, La mente consciente (Gedisa, Barcelona 1999), p.163

(20) Ver, por ejemplo, Stuart R.Hemeroff y Roger Penrose, "Orchestated reduction of quantum coherence in brain microtubules: a model of consciousness", en Toward a Science of Consciousness, S.R. Hameroff, A. W. Kaszniak y A.C Scott, eds. (The MIT Press, Cambridge, Mass. 1996), pp. 507-540.

* Catedrático de Historia de la Ciencia en el Departamento de Física Teórica de la Universidad Autónoma de Madrid

Teléf. 913974877 - Fax 913973936

Fecha de recepción: 04-09-02 